\title{
Liens transnationaux et santé mentale : de la nécessité du lien entre ici et là-bas? Le cas des migrations africaines en Île-de-France
}

Transnational Ties and Mental Health: A Need for a Link between Here and There? The Case of African Migrations in France

Vínculos trasnacionales y salud mental: ¿necesidad de una conexión aquí y allá?

El caso de las migraciones africanas en Francia

Julie Pannetier

\section{OpenEdition}

\section{Journals}

Édition électronique

URL : https://journals.openedition.org/remi/10817

DOI : 10.4000/remi. 10817

ISSN : $1777-5418$

Éditeur

Université de Poitiers

Édition imprimée

Date de publication : 1 octobre 2018

Pagination : 79-99

ISBN : 979-10-90426-62-7

ISSN : 0765-0752

Référence électronique

Julie Pannetier, « Liens transnationaux et santé mentale : de la nécessité du lien entre ici et là-bas ? Le cas des migrations africaines en Île-de-France », Revue européenne des migrations internationales [En ligne], vol. 34 - n² et $3 \mid 2018$, mis en ligne le 01 janvier 2021, consulté le 15 avril 2022. URL : http:// journals.openedition.org/remi/10817; DOI : https://doi.org/10.4000/remi.10817 


\title{
Liens transnationaux et santé mentale : de la nécessité du lien entre ici et là-bas? Le cas des migrations africaines en Île-de-France
}

\author{
Julie Pannetier ${ }^{1}$
}

Les personnes ayant entrepris une migration maintiennent souvent des liens avec leur pays d'origine. Certains débats politiques et scientifiques portent d'ailleurs sur l'entrave que représenteraient ces liens transnationaux, et les pratiques transnationales qui en découlent, à l'insertion sociale des personnes dans leur nouveau pays de résidence. Cependant, l'enquête Trajectoires et Origines 2008 a permis de souligner que les pratiques transnationales étaient plutôt le fait des personnes les plus insérées socialement alors que celles qui étaient dans l'attente d'un titre de séjour se trouvaient davantage en situation de rupture avec leur pays d'origine (Beauchemin et al., 2015b). La question du rôle des pratiques transnationales sur la santé des populations fait également l'objet de débats scientifiques, surtout aux États-Unis à propos des populations latino-américaines immigrées. Leurs pratiques transnationales représenteraient une source de stress délétères pour leur santé physique et mentale, mais constitueraient aussi une source de résilience en réaction à un ensemble de difficultés rencontrées aux États-Unis, telles que l'isolement social et les discriminations (Torres et al., 2016a). L'enquête Parcours 2012-2013, première étude de grande ampleur portant spécifiquement sur les migrations subsahariennes et la santé, permet d'analyser le rôle de certains liens transnationaux sur la santé mentale des

1 Démographe, Maîtresse de Conférences à I'Université Paris Nanterre, Cresppa-GTM, Chercheure associée au Ceped-SageSud et Fellow de I'Institut des Migrations,

200 avenue de la République, 92001 Nanterre cedex; julie.pannetier@ parisnanterre.fr Cet article a été écrit par l'auteure pour le groupe Parcours qui est composé de Annabel Desgrées du Loû, France Lert, Rosemary Dray Spira, Nathalie Bajos, Nathalie Lydié (responsables scientifiques), Julie Pannetier, Andrainolo Ravalihasy, Anne Gosselin, Elise Rodary, Dolores Pourette, Joseph Situ, Pascal Revault, Philippe Sogni , Julien Gelly, Yann Le Strat, Nicolas Razafindratsima.

L'auteure remercie Annabel Desgrées du Loû pour sa relecture d'une version préliminaire de ce texte ainsi que les évaluateurs anonymes et les éditrices de ce numéro pour leurs remarques et suggestions. Elle remercie les personnes qui ont accepté de participer à I'enquête ANRS-PARCOURS, les associations qui ont soutenu cette étude : SOS Hépatites (Michelle Cizorne), le Comede (Pascal Revault, Arnaud Veïsse, Laurence Wolmark), le RAAC-SIDA et le FORIM (Jean-Charles Ahomadegbe, Khady Sakho). Ses remerciements vont aussi à Géraldine Vivier (INED), Eva Lelièvre (INED) et Anne Gervais (AP-HP) pour leur contribution à la préparation des questionnaires, à Henri Panjo pour son aide à la méthodologie statistique, ainsi qu'à Agnès Guillaume (IRD) pour la communication sur I'enquête. Ce travail a été réalisé grâce à une allocation postdoctorale de I'ANRS. 
populations africaines immigrées résidant en Île-de-France et de contribuer ainsi à ce débat. Nous présenterons d'abord ce qu'implique l'approche transnationale pour l'étude de la santé des populations migrantes. Puis, deux aspects des liens transnationaux; la parentalité transnationale et le soutien apporté par les proches restés au pays, seront mis en relation avec les symptômes anxieux et dépressifs ressentis par les personnes enquêtées. Ces faits sociaux sont traduits par des indicateurs et des échelles de mesure et cela implique, au préalable, une réflexion méthodologique.

\section{Santé et migrations : vers une approche transnationale?}

\section{La santé mentale en migration}

Plusieurs études épidémiologiques européennes indiquent que les niveaux de dépression sont plus élevés parmi les populations immigrées que parmi les populations majoritaires ${ }^{2}$ (Bhui et al., 2005; Levecque et al., 2007; Missinne et Bracke, 2012), du fait notamment des inégalités socioéconomiques (accès à l'emploi, niveau de revenu) et des discriminations liées à l'origine. À l'échelle du pays, à ce jour, aucune étude en France ne rend compte des inégalités de santé en lien avec le statut migratoire et/ou l'origine pour les affections mentales les plus fréquentes : la dépression et l'anxiété. La caractéristique française d'interdiction des statistiques ethniques a contribué à invisibiliser ces disparités. Le débat qui a porté sur les statistiques ethniques a encouragé de nouvelles pratiques de recueil de données (Fassin et Fassin, 2006). Cependant, les grandes enquêtes statistiques nationales ${ }^{3}$ ont permis $d^{\prime}$ analyser uniquement les disparités concernant l'état général de santé perçu des populations. Les populations immigrées se perçoivent en plus mauvaise santé que la population majoritaire, en raison d'inégalités à la fois liées au capital social (réseau de soutien), au capital économique (revenu et catégorie socioprofessionnelle) (Berchet et Jusot, 2010) et aux discriminations (Hamel et Moisy, 2012). Mais l'état général de santé perçu n'est pas superposable à l'état de santé mentale des populations.

Des inégalités de santé mentale en défaveur des populations immigrées ont bien été repérées néanmoins à des échelles plus réduites. Par exemple, les services hospitaliers psychiatriques du $20^{\mathrm{e}}$ arrondissement de Paris accueillent proportionnellement ${ }^{4}$ plus de personnes nées en Afrique subsaharienne que de personnes nées en France. Les conditions de vie difficiles de cette population en Île-de-France et les discriminations qu'elles subissent pourraient expliquer cette surreprésentation (Tortelli et al., 2014). Toujours en Île-de-France, l'enquête Parcours réalisée auprès de populations africaines immigrées suivies dans des structures de soin, atteste également de niveaux de symptômes anxieux et dépressifs très élevés en raison notamment de l'insécurité résidentielle et

2 Le terme "population majoritaire" désigne l'ensemble des personnes, numériquement les plus nombreuses, qui résident dans les pays concernés et qui ne sont ni immigrées ni descendantes de personnes immigrées.

3 Les enquêtes Santé Protection Sociale 2006 (Berchet et Jusot, 2010) et Trajectoire et Origine 2008 (Hamel et Moisy, 2012) par exemple.

4 C'est-à-dire en tenant compte de leur représentation respective dans le $20 \mathrm{e}$ arrondissement de Paris. 
administrative à l'arrivée en France. Les processus qui portent atteinte à la santé mentale des femmes et des hommes sont différents du fait d'une vulnérabilité genrée à différents types de violences (Pannetier et al., 2017). Les troubles psychiques sont également fréquents chez les personnes accompagnées par le Comité pour la santé des exilés (Comede) tout comme différentes formes de violences et de vulnérabilités sociales (Veïsse et al., 2017).

Par ailleurs, des travaux sociologiques et anthropologiques sur la santé mentale en migration en France portent notamment sur la sociogenèse des souffrances psychiques, c'est-à-dire sur les conditions sociales d'émergence de troubles mentaux, analysées en lien avec la mobilité sociale et intergénérationnelle (Sayad, 2006; Wang, 2017) et sur la place de "culture" dans la prise en charge des affections mentales (Wang, 2016). Sur ce point, le culturalisme de certaines approches cliniques a été souligné. Considérer les personnes migrantes avant tout comme "des êtres de culture autre" devant bénéficier d'un traitement différent peut conduire à l'organisation de soins spécifiques inadaptés à leur pathologie (Rechtman, 2000; Fassin, 2000). Les recherches ayant une démarche statistique ont par conséquent un rôle important à jouer, en particulier dans l'analyse quantitative des déterminants des affections mentales des populations, puisqu'elles contribuent à mieux les comprendre et à améliorer ainsi leur prise en charge. Les effets des liens transnationaux sur la santé mentale des populations migrantes sont complexes, encore mal connus et dépendent des configurations familiales à distance et des positions des personnes dans les rapports sociaux.

\section{Liens transnationaux et santé mentale : facteurs de risque ou de résilience?}

On ne peut évoquer les liens que les personnes migrantes maintiennent avec leur pays d'origine sans mentionner l'approche transnationale, née au début des années 1990 aux États-Unis, sous la plume de trois anthropologues: Glick Schiller, Basch et Szanton Blanc (1995). Les personnes migrantes ont des relations sociales multiformes qui relient leur pays d'origine et leur pays de résidence. Ainsi, l'approche transnationale implique non plus l'idée d'un "ici» ou "là-bas", mais plutôt celle d'un «ici et là-bas" articulé (Razy et Baby-Collin, 2011). L'approche transnationale s'inscrit, en ce sens, en opposition à un système de référencement (culturel, identitaire et politique) fondé sur une division du monde en États-Nation. Le transnationalisme renvoie ainsi à des pratiques transnationales, non institutionnelles ou étatiques, mises en place par les personnes, et constitue ainsi une forme de "mondialisation par le bas" (Portes, 1999). Plusieurs travaux décrivent les dimensions économique (transfères monétaires, création d'entreprises transnationales), politique (participation à la vie politique du pays d'origine), ou socioculturelle (participation à des carnavals populaires) que peuvent prendre ces pratiques (Levitt et Jaworsky, 2007).

L'approche transnationale implique donc de prendre en compte la multiplicité des univers d'identification des personnes migrantes et de pouvoir repérer empiriquement la fréquence et l'intensité des liens et des pratiques transnationales. Comme les personnes migrantes peuvent être inscrites dans des logiques de dettes matérielles, sociales ou affectives vis-à-vis de leur famille (Ly et al., 2014), la question du coût des pratiques transnationales pour leur 
santé, notamment des remises ${ }^{5}$, a été soulignée. Des études quantitatives, en épidémiologie sociale, ont été réalisées aux États-Unis auprès des populations latino-américaines afin de mesurer l'effet des pratiques transnationales sur la santé mentale de ces dernières (Torres et al., 2016a). Ces recherches montrent que certaines pratiques transnationales, notamment lorsqu'elles impliquent des transferts monétaires ou des retours ponctuels dans le pays d'origine, peuvent représenter une source de stress et entrainer un fardeau social et financier pour les personnes. Cela dit, l'effet des liens transnationaux sur la dépression par exemple dépend étroitement de la position sociale des personnes. Les femmes semblent plus affectées par les remises et les allers et retours entre le pays d'origine et le pays de résidence, possiblement en raison d'attentes plus fortes les concernant, en termes de soutien financier alors qu'elles sont souvent moins en capacité d'y répondre ou parce qu'elles doivent jouer un double rôle d'aidantes auprès de leurs proches, à la fois dans le pays d'origine et dans celui où elles ont émigré (Alcántara et al., 2015; Torre, et al., 2016b; Torres et al., 2016a).

Depuis les années 2000, la famille à distance ou transnationale fait l'objet de recherches dans le champ des migrations internationales (Audebert et al., 2018). Les stratégies migratoires impliquent souvent une réciprocité des échanges transnationaux, lorsque par exemple les personnes migrantes sont les pourvoyeuses de remises pour la famille, pendant que les grands-parents ou d'autres membres de la parenté ont en garde leurs enfants restés au pays. Le "soutien" et la "dépendance" dans les familles transnationales ne sont donc pas des pratiques distinctives et qui s'opposent, elles peuvent se superposer (Razy et Baby-Collin, 2011). La parentalité transnationale a fait l'objet d'investigations sur la nature des arrangements familiaux, sur les pratiques visant à maintenir les relations à distance, et plus récemment sur le travail quotidien nécessaire au maintien de relations transnationales entre les parents migrants et les parents ou les personnes qui en en garde les enfants (Poeze et al., 2016).

La parentalité transnationale est souvent considérée comme une source de souffrance pour les personnes immigrées et leurs enfants restées au pays. D'après certaines enquêtes quantitatives, les populations latino-américaines immigrées aux États-Unis semblent être affectées par cette séparation, qu'il s'agisse des parents (Ornelas et Perreira, 2011) ou des jeunes adultes séparés de leurs parents pendant l'enfance (Torres, 2013). Une enquête qualitative sur les migrations entre la Bolivie et l'Italie et la maternité transnationale relate, par exemple, que le rôle de mère à distance peut être source de souffrances et de conflits concernant l'éducation des enfants avec le père ou la grand-mère ayant la garde des enfants (Yépez et al., 2011). Cela dit, vivre à distance n'entraine pas systématiquement un "mal-être" pour les parents migrants et celui-ci est largement dépendant de la situation administrative et sociale de ces derniers (Haagsman et al., 2015). Des chercheures ont souligné les différentes stratégies permettant aux parents nigérians vivant aux Pays-Bas d'atténuer les tensions liées à la séparation d'avec leurs enfants, dont le maintien d'un sentiment fort d'appartenance (Berckmoes et Mazzucato, 2018). Enfin, au Ghana, les jeunes de douze à vingt-et-un ans dont le père, la mère ou les deux parents sont absents et dont la garde est confiée à un parent ou un proche rapportent des niveaux de

5 Les remises sont des transferts monétaires des personnes migrantes vers leur pays d'origine. 
bien-être aussi élevés voir plus élevés que les enfants de familles non migrantes (Cebotari, Mazzucato et Appiah, 2018).

Les liens transnationaux qui unissent les personnes migrantes et non migrantes par des liens de parenté, d'amitiés ou d'origine communautaire partagés constituent également une forme de capital social. Pour Massey et al. (1993), les pratiques transnationales sont liées à la condition d'infériorisation des personnes immigrées dans leurs sociétés d'immigration. Des anthropologues américaines suggèrent également que les discriminations dont les enfants des personnes immigrées sont la cible aux États-Unis constituaient une motivation pour rester en contact avec la société d'origine de leurs parents (Fouron et Schiller, 2002). Cette observation s'avère également vraie à une échelle plus large en France, les pratiques transnationales des descendant.e.s d'immigrée.es sont plus fréquentes parmi celles et ceux qui déclarent avoir subi des discriminations en raison de leur origine (Beauchemin et al., 2015b). Les liens transnationaux offrent ainsi possiblement un espace social dans lequel leur condition n'est pas minoritaire et où elles n'ont pas à souffrir du racisme. Deux études qualitatives, anthropologiques, I'une conduite à Paris auprès des personnes maliennes et I'autre à Détroit après de Mexicaines font référence à l'effet bénéfique des liens et échanges transnationaux offrant "un sentiment d'appartenance" (Sargent et Larchanché, 2016); "an alternative space of belonging" (Viruell-Fuentes et Schulz, 2009) permettant de pallier un ensemble de difficultés vécues dans le pays de résidence, telles que l'isolement social et les discriminations. Les discriminations sont d'ailleurs largement rapportées par les populations africaines immigrées en France (Beauchemin et al., 2010).

\section{Migrations africaines et liens transnationaux}

\section{Les migrations africaines en France}

L'immigration subsaharienne en France, bien qu'existant aux XIXe et XXe siècles ${ }^{6}$, prend véritablement son essor dans les années 1970. Les personnes immigrées d'Afrique subsaharienne étaient 20000 en 1962 et 570000 en 2004. En 2004, les personnes immigrées nées en Afrique subsaharienne représentaient $12 \%$ des immigré.e-s vivant en France et $17 \%$ des entrées d'immigrant.e-s dans le pays (Lessault et Beauchemin, 2009). En 2011, elles représentaient $1 \%$ de la population résidant en France selon le recensement de la population (INSEE, 2011).

Jusqu'au début des années 1970, les migrations subsahariennes en France étaient essentiellement masculines et économiques, et elles provenaient des anciennes colonies françaises de la vallée du fleuve Sénégal (Mali, Sénégal, Mauritanie). II s'agissait de migrations temporaires qualifiées de "migrations tournantes" ou de "noria migratoire", fonctionnant sur un système collectif (familiale et/ou communautaire) de rotation où les cadets remplaçaient leurs ainés, les immigrés occupaient pour un temps les emplois peu qualifiés du secteur de I'industrie automobile notamment (Timera et Garnier, 2010). À partir

6 II s'agit notamment de navigateurs installés notamment dans le port de Marseille ou des soldats autrement appelés les "tirailleurs sénégalais" restés en France (Ndiaye, 2008). 
du milieu des années 1970, sous l'effet de la fermeture des frontières à l'immigration de travail extra-européenne en France et dans un contexte de paupérisation du Sahel, la dynamique migratoire en provenance d'Afrique subsaharienne s'est transformée. Les migrations temporaires sont devenues durables, puisque les allers et retours entre le pays d'origine et la France étaient devenus impossibles. Les stratégies migratoires se sont diversifiées incluant les migrations réalisées pour faire des études, pour fuir une instabilité économique et/ou politique ou pour "tenter l'aventure" (Bredeloup, 2008). Impulsée par le changement de politique migratoire favorisant le regroupement familial au détriment de l'immigration de travail, I'immigration féminine a augmenté de $177 \%$ entre 1975 et 1982 (Couillet, 2010). Les migrantes arrivent fréquemment seules afin de rechercher un emploi, étudier ou fuyant des persécutions/menaces dans leur pays (Beauchemin et al., 2013; Gosselin et al., 2016). Les logiques migratoires des femmes et des hommes se rapprochent. En 2011, les femmes représentaient $51 \%$ des personnes originaires d'Afrique subsaharienne résidant en France (INSEE, 2011).

Malgré un niveau d'éducation élevé (Ichou et al., 2017), les conditions de vie des migrants subsahariens en France apparaissent particulièrement difficiles. Les femmes et des hommes immigrés d'Afrique subsaharienne sont particulièrement touchés par l'inactivité et le chômage (Meurs et al., 2007) et lorsqu'ils travaillent, leur salaire horaire est en moyenne de $15 \%$ inférieur à celui de la population majoritaire (Meurs et al., 2015). Par ailleurs, des difficultés sociales peuvent s'étendre sur une longue période puisqu'il faut respectivement six et sept ans après l'arrivée en France pour que la moitié des femmes et des hommes obtiennent une stabilité minimale, c'est-à-dire un titre de séjour d'au moins un an, un logement personnel et une activité permettant d'être financièrement indépendant $^{7}$ (Gosselin et al., 2016).

\section{Les liens transnationaux entre les populations africaines et leurs pays d'origine}

L'enquête Trajectoires et Origines 2008 a permis de mesurer le transnationalisme, faisant référence à un ensemble de pratiques ou d'orientations sociales, économiques et politiques conduites en dehors des frontières du pays de résidence (Beauchemin et al., 2015b). À partir d'une dizaine de questions, des informations sur des pratiques transnationales dans quatre grands domaines, économique, politique, symbolique et social, ont été collectées. Les personnes originaires du Sahel ont plus de pratiques transnationales que les personnes originaires d'Asie du Sud-Est et de manière générale des personnes originaires d'Afrique subsaharienne se caractérisent par un fort transnationalisme économique : elles sont les plus susceptibles de participer aux transferts financiers, elles invertissent davantage dans leur pays d'origine, elles y sont plus souvent propriétaires et participent plus à des investissements collectifs. Le transnationalisme social est également important : elles ont des contacts réguliers avec leur famille hors de France et elles retournent souvent dans leur pays d'origine (Beauchemin et al., 2015b).

7 Ces temps d'installation ne concernent ni les étudiants ni les personnes qui résident hors région parisienne (Gosselin et al., 2016). 
Par ailleurs, les structures sociales sont organisées de façon à ce que la communauté et la famille élargie restent les unités de base où la solidarité est maintenue (Findley, 1999). En effet, surtout pour les populations originaires de la région du Sahel, l'organisation des structures sociales favorise le maintien des liens par-delà les frontières, les personnes immigrées sont impliquées dans l'organisation collective pour la construction d'école, de centre de santé, etc. Par ailleurs, la migration est aussi une "stratégie" de diversification des revenus familiaux et communautaires, grâce à laquelle les migrants sont "envoyés" et sont supposés, implicitement, contribuer en retour à l'économie de la famille et de la communauté (Quiminal, 1991).

En Europe, la part des personnes immigrées originaires d'Afrique subsaharienne dont le conjoint ou un enfant est resté au pays varie selon le pays de naissance et l'ancienneté de la migration. Le regroupement familial semble plus fréquent dans les pays où les migrations sont plus anciennes. Les possibilités de réunification familiale après la migration et les retours ponctuels ou la visite des enfants dépendent étroitement des situations économique et administrative des personnes (Beauchemin et al., 2014; Mazzucato et al., 2015).

\section{La mesure des liens transnationaux et de la santé mentale : réflexions méthodologiques}

Les résultats présentés ici sont issus des données de l'enquête Parcours menée en 2012-2013 dans soixante-quatorze structures de soin en Île-de-France auprès de 2468 personnes immigrées d'Afrique subsaharienne (Desgrées du Loû et Lert, 2017). Deux aspects des liens transnationaux peuvent être mis en relation avec la santé mentale des personnes au moment de l'enquête : la parentalité transnationale et le soutien transnational. Ces dimensions sont traduites par des indicateurs dont il s'agit de discuter la valeur heuristique. Ils ont été définis a posteriori, l'enquête Parcours n'ayant pas pour objectif d'analyser la problématique des liens transnationaux en lien avec la santé.

\section{L'enquête Parcours}

En France, les populations originaires d'Afrique subsaharienne étant particulièrement touchée par le $\mathrm{VIH} / \mathrm{sida}^{8}$ et I'hépatite $\mathrm{B}$ chronique, I'enquête Parcours a été menée pour comprendre dans cette population les déterminants sociaux de l'exposition à cette pathologie ainsi que les facteurs facilitant ou faisant obstacle à sa prise en charge et à son traitement. II s'agit d'une enquête biographique qui retrace l'ensemble du parcours de vie des personnes, sous forme de différentes trajectoires de vie : affective et familiale, professionnelle, l'itinéraire migratoire, les logements et les titres de séjours successifs. L'idée est de pouvoir analyser I'enchainement des situations de vie, pour comprendre quand et comment l'infection $\mathrm{VIH} /$ sida s'inscrit dans le parcours de vie. L'enquête visait aussi à replacer dans le parcours biographique I'histoire des dépistages et des diagnostics du $\mathrm{VIH}$ et de I'hépatite $\mathrm{B}$, I'histoire de l'accès aux soins et à déterminer ce que ces deux pathologies changent dans le parcours de vie, d'où le titre de l'enquête

8 En 2013, les personnes immigrées originaires d'Afrique subsaharienne représentaient $31 \%$ des nouveaux diagnostics $\mathrm{VIH}$ ( $61 \%$ chez les femmes et $17 \%$ chez les hommes) (Cazein et al., 2015). 
"Parcours" (Encadré 1). Cette approche biographique, largement utilisée par les sociologues qui s'appuient sur le récit de vie afin de comprendre une situation à un moment donné, demeure récente dans les sciences sociales quantitatives (Antoine et al., 2006).

\title{
Encadré 1 : L'enquête Parcours: une étude sur le VIH, l'hépatite B et la santé dans les parcours de vie des immigrés subsahariens vivant en Île-de-France
}

\begin{abstract}
L'enquête ANRS Parcours a été conduite entre février 2012 et mai 2013 dans soixante-quatorze structures de santé d'île-de-France. Elle a interrogé un échantillon représentatif de trois groupes de personnes immigrées originaires d'Afrique subsaharienne : un groupe de 926 personnes vivant avec le $\mathrm{VIH}$, un groupe de 779 personnes porteuses d'une hépatite $B$ chronique et un groupe de 763 personnes n'ayant aucune de ces deux pathologies et recrutées dans des centres de santé de médecine générale. Étaient éligibles les personnes âgées de dix-huit à cinquanteneuf ans résidant en Île-de-France et nées dans un pays d'Afrique subsaharienne et de nationalité de l'un de ces pays à la naissance, quelle que soit la nationalité au moment de l'enquête. L'enquête était proposée aux personnes éligibles par leur médecin au cours de la consultation. L'entretien était réalisé par un enquêteur spécialisé, soit à l'issue de la consultation médicale, soit sur rendez-vous. Toutes les informations ont été recueillies de façon anonyme. Comme $60 \%$ des personnes immigrées originaires d'Afrique subsaharienne en France vivent en Île-de-France, cette enquête renseigne sur la situation de la majorité des personnes originaires d'Afrique subsaharienne en France. Plus d'information sur www.parcours-santemigration.com et sur http://www.ceped.org/parcours/ L'enquête PARCOURS a été financée par l'Agence nationale de recherche contre le sida et les hépatites virales (ANRS), avec le soutien de la Direction générale de la Santé. Le recueil des données a été assuré par les sociétés ClinSearch et Ipsos.
\end{abstract}

Cette enquête a été réalisée dans des structures de santé accessibles à toute personne, sans condition de titre de séjour ou de revenus, et constituant des espaces où la confidentialité relative à la maladie et aux conditions de séjour est garantie. Elle a été réalisée dans des services hospitaliers de maladies infectieuses où sont suivies les personnes pour une infection VIH et dans diverses structures de santé de prise en charge de l'hépatite B. En outre, cette enquête a été menée auprès de personnes n'ayant aucune de ces deux pathologies, d'une part pour décrire et comprendre les situations vécues par les personnes immigrées originaires d'Afrique subsaharienne, indépendamment de ces deux maladies. L'enquête auprès de ce groupe, que nous appellerons groupe de référence par la suite, a été réalisée lors des consultations en médecine générale dans des centres de santé, y compris dans des dispositifs de soins s'adressant aux populations les plus précaires. La réussite de cette d'enquête est d'être parvenue à toucher des personnes sans titre de séjour qui sont habituellement difficiles à contacter dans les enquêtes en population générale. Par contre, sa limite est qu'elle n'atteint pas les personnes qui ne consultent jamais dans les structures de santé enquêtées comme les plus jeunes qui sont en meilleure santé et donc consultent un peu moins en structures de soins. 


\section{Tableau 1 : Caractéristiques sociodémographiques des femmes et des hommes selon le groupe d'étude}

\begin{tabular}{|c|c|c|c|c|c|c|}
\hline & \multicolumn{3}{|c|}{ Femmes } & \multicolumn{3}{|c|}{ Hommes } \\
\hline & $\begin{array}{l}\text { Groupe de } \\
\text { référence }\end{array}$ & VIH & Hépatite B & $\begin{array}{l}\text { Groupe de } \\
\text { référence }\end{array}$ & VIH & Hépatite $B$ \\
\hline & $N=405$ & $N=568$ & $N=216$ & $N=351$ & $N=349$ & $N=553$ \\
\hline & Med/\% & Med/\% & Med/\% & Med/\% & Med/\% & Med/\% \\
\hline \multicolumn{7}{|c|}{ Caractéristiques sociodémographiques } \\
\hline Âge médian & 41 & 40 & 37 & 41 & 47 & 39 \\
\hline $\begin{array}{l}\text { Niveau d'éducation } \\
\text { secondaire/supérieur }\end{array}$ & 80,1 & 81,2 & 78,6 & 76,1 & 80,1 & 68,1 \\
\hline \multicolumn{7}{|l|}{ Situation professionnelle } \\
\hline $\begin{array}{l}\text { Emploi qualifications } \\
\text { faibles/« petits boulots» }\end{array}$ & 39,4 & 42,4 & 33,5 & 40 & 44,2 & 49,2 \\
\hline $\begin{array}{l}\text { Emploi qualifications } \\
\text { intermédiaires/élevées }\end{array}$ & 22,0 & 16,0 & 17,2 & 26,2 & 28,2 & 25,3 \\
\hline Inactif & 33,1 & 35,0 & 38,1 & 27,0 & 26,1 & 21,9 \\
\hline Étudiant & 5,5 & 6,6 & 11,1 & 6,9 & 1,6 & 3,7 \\
\hline Être en couple & 51,1 & 33,6 & 45,8 & 38,1 & 34,6 & 38,4 \\
\hline \multicolumn{7}{|c|}{ Caractéristiques liées à la migration } \\
\hline $\begin{array}{l}\text { Durée médiane de } \\
\text { séjour en France }\end{array}$ & 13 & 12 & 10 & 12 & 13 & 12 \\
\hline $\begin{array}{l}\text { Avoir vécu } 7 \text { ans ou } \\
\text { plus en France }\end{array}$ & 67,8 & 69,5 & 53,9 & 61,4 & 73,2 & 62,5 \\
\hline \multicolumn{7}{|l|}{ Raison de la migration } \\
\hline $\begin{array}{l}\text { Menacée dans } \\
\text { son pays }\end{array}$ & 17,3 & 9,8 & 13,7 & 24,1 & 17,9 & 17,6 \\
\hline Raisons de santé & 3,7 & 8,4 & 7,0 & 1,8 & 9,2 & 5,8 \\
\hline $\begin{array}{l}\text { Tenter sa chance/ } \\
\text { trouver du travail }\end{array}$ & 19,8 & 35,2 & 25,2 & 37,8 & 42,9 & 48,1 \\
\hline Études & 13,0 & 8,1 & 6,8 & 20,6 & 15,4 & 16,5 \\
\hline Rejoindre de la famille & 46,1 & 38,6 & 47,3 & 15,7 & 14,6 & 11,9 \\
\hline \multicolumn{7}{|c|}{ Situation administrative à l'enquête } \\
\hline $\begin{array}{l}\text { Autorisation provisoire/ } \\
\text { pas de titre de séjour }\end{array}$ & 13,8 & 6,9 & 21,8 & 20,9 & 10,2 & 25,8 \\
\hline $\begin{array}{l}\text { Carte de séjour } \\
\text { temporaire ( } 1 \text { an) }\end{array}$ & 24,1 & 42,5 & 33,1 & 27,2 & 44,6 & 36,7 \\
\hline $\begin{array}{l}\text { Nationalité française/ } \\
\text { carte de résident } \\
\text { (10 ans) }\end{array}$ & 62,1 & 50,5 & 45,0 & 51,9 & 45,3 & 37,5 \\
\hline
\end{tabular}

Source : Étude ANRS-Parcours 2012-2013. 
Les données biographiques de cette enquête, combinées à des données biomédicales collectées par les médecins ont notamment permis de montrer que le poids de l'épidémie de $\mathrm{VIH} /$ sida dans les populations originaires d'Afrique subsaharienne et vivant en France n'était pas seulement lié à la prévalence dans les pays d'origine, mais qu'il y avait un risque d'infection en France (Desgrées du Loû et al., 2015). Cette étude met également au jour les facteurs structurels qui pèsent, souvent de façon durable, sur l'installation des immigrés en France et accroissent le risque d'être infecté par le VIH une fois sur place (Desgrées du Loû et al., 2016).

Les trois groupes de personnes enquêtées : les personnes vivant avec le $\mathrm{VIH}$, les personnes porteuses d'une hépatite B chronique et les personnes n'ayant aucune de ces deux pathologies forment une population très diversifiée et dont les caractéristiques démographiques correspondent bien à ce que l'on sait des migrations subsahariennes en France. Les personnes enquêtées sont très majoritairement originaires d'anciennes colonies françaises, les pays les plus représentés étant le Mali, la Côte d'Ivoire et le Sénégal pour Afrique de l'Ouest et le Congo-Brazzaville et la République Démocratique du Congo et le Cameroun pour Afrique de l'Est. L'âge moyen est de quarante-et-un ans, légèrement plus élevé que dans I'enquête Trajectoires et Origines 2008, du fait du lieu de recrutement. La principale raison de la migration pour les femmes était le regroupement familial $(46 \%)$ et le fait de chercher un emploi ou "tenter leur chance" pour les hommes (38\%). Cela dit les femmes migrent aussi souvent seules et leurs situations migratoires se diversifient. Les situations par rapport au titre de séjour sont très contrastées. La part des femmes et des hommes qui se déclarent sans titre de séjour au moment de l'enquête est très élevée (respectivement $14 \%$ et $21 \%$ dans le groupe de référence), mais la majorité des femmes et des hommes avaient un titre de séjour long ou l'acquisition de la nationalité française (respectivement $52 \%$ et $62 \%$ ).

\section{Définir la parentalité et le soutien transnational}

Les mouvements migratoires peuvent séparer les membres d'une même famille dont les membres peuvent résider dans plusieurs pays, tout en maintenant des relations fortes et suivies dans le temps et à distance. L'enquête Parcours permet d'explorer une partie du questionnement lié à la famille à distance; celle de la parentalité transnationale et de son implication pour la santé mentale des personnes ayant réalisé une migration. Cela concerne ici les personnes dont au moins un enfant réside dans le pays d'origine ou dans un autre pays. Pour cette étude, nous avons considéré uniquement les enfants de moins de dix-huit ans, toujours éligibles au regroupement familial ${ }^{9}$. Cet indicateur est très solide sur le plan méthodologique dans la mesure où les informations sont recueillies de manière factuelle. Les informations sont recueillies à travers l'outil de collecte des enquêtes biographiques rétrospective, une fiche AGEVEN ou une grille biographique. II était tout d'abord demandé aux personnes de déclarer l'année de naissance de leurs enfants, cette information est située dans le parcours de vie (elle est reportée sur la grille biographique). Puis les personnes renseignaient le lieu de vie de leurs enfants au moment de l'enquête. Les personnes

9 Les résultats des analyses sont similaires lorsque I'on considère uniquement les enfants de moins de quinze ans. 
ne répondaient donc pas directement à une question du type «Un de vos enfants réside-t-il à l'étranger?", ce qui pourrait entrainer un biais de déclaration dans la mesure où les personnes peuvent percevoir le stigmate associé au fait d'avoir un enfant mineur au pays. Les hommes ont déclaré deux fois plus souvent que les femmes avoir un enfant de moins de dix-huit ans à l'étranger : $33 \%$ contre $15 \%$ dans le groupe de référence (Tableau 2). II n'y avait pas de différence entre les trois groupes d'étude sur ce point.

La migration n'implique pas nécessairement une rupture avec les personnes restées au pays, des liens transnationaux subsistent et recouvrent des dimensions multiples : politique, économique et sociale. L'enquête Parcours permet de questionner une dimension subjective du lien transnational : le sentiment d'avoir quelqu'un sur qui compter dans son pays d'origine. Cet indicateur découle de la question suivante : "Actuellement, avez-vous dans votre entourage quelqu'un sur qui vous pouvez compter dans les moments difficiles?". Les personnes pouvaient répondre : oui en France, oui dans le pays d'origine ou dans un autre pays (plusieurs réponses étaient possibles) ou non. Cette question visait à caractériser le réseau social des personnes qui ont le VIH, I'hépatite B ou ni l'une ni l'autre de ces deux pathologies, afin d'évaluer l'effet possible de la maladie sur l'isolement social. Les modalités de ce soutien n'ont pas été questionnées. Comme tout indicateur subjectif, déclarer avoir quelqu'un sur qui compter dans les moments difficiles, n'informe pas sur la réalité de ce soutien. Cet indicateur ne permet pas de questionner le sens et la valeur des liens transnationaux qui peuvent varier selon la position sociale des personnes et selon les conditions de leur migration. Cela dit déclarer pouvoir compter sur quelqu'un dans le pays d'origine signifie d'une part que la personne a des liens transnationaux et d'autre part que ces liens sont a priori mobilisables. Dans la majorité des cas, les personnes déclarent avoir quelqu'un sur qui compter à la fois dans le pays d'origine et en France ( $72 \%$ des femmes et $65 \%$ des hommes du groupe de référence; tableau 2). Seuls 8 à $13 \%$ des personnes interrogées selon leur sexe et leur pathologie déclarent n'avoir personne sur qui compter dans les moments difficiles. Les femmes ayant le VIH déclarent moins fréquemment avoir quelqu'un sur qui compter dans le pays d'origine possiblement en raison d'un éloignement dû à la crainte de la stigmatisation associée au VIH.

\section{Mesurer les symptômes anxieux et dépressifs}

L'enquête Parcours ne porte pas spécifiquement sur la santé mentale, mais elle comporte un jeu de quatre questions : le "Patient Heath Questionnaire-4" (PHQ-4) permettant de calculer un score de symptomatologie anxieuse et/ou dépressive (Kroenke et al., 2009). Le PHQ-4 est initialement un outil permettant d'indiquer une symptomatologie anxieuse et/ou dépressive modérée à sévère méritant une évaluation plus approfondie par un diagnostic clinique. Concrètement, les personnes interrogées devaient rapporter à quelle fréquence durant les deux dernières semaines, elles avaient été gênées par les problèmes suivants : 1 . un sentiment de nervosité, d'anxiété ou de tension; 2 . une incapacité à arrêter de s'inquiéter ou à contrôler ses inquiétudes; 3 . peu d'intérêt ou de plaisir à faire les choses; 4. être triste, déprimée ou désespérée. Les réponses à ces questions pouvaient être : jamais, plusieurs jours, plus de la moitié du temps ou presque tous les jours, auxquelles sont associées des points de 0 à 3. Les personnes sont considérées comme ayant des symptômes anxieux et 
dépressifs si elles totalisent plus de 6 points sur cette échelle variant de 0 à 12, ce qui correspond à une symptomatologie anxieuse et/ou dépressive modérée à sévère (Kroenke et al., 2009).

Les niveaux de symptômes anxieux et dépressifs observés dans cette étude ne sont pas assimilables à des troubles anxieux et dépressifs tels qu'ils pourraient être repérés par un diagnostic clinique. Le PHQ-4 prend la mesure d'une subjectivité individuelle. II faut se reconnaitre dans les items proposés pour les déclarer. De plus, les échelles de mesure standardisées comme le PHQ-4 sont la plupart du temps conçues avec des notions occidentales de la maladie et de ces symptômes, l'utilisation de ces instruments pour des populations qui ont d'autres normes sociales d'expression de ces symptômes que celles pour qui elles ont été produites demanderait donc une validation préalable. Des échelles de mesure questionnant plus directement les symptômes physiques et la somatisation permettraient d'éviter les sous-déclarations lors de l'évaluation des souffrances psychiques (Beutel et al., 2016). Ceci précisé, les niveaux de symptômes anxieux et dépressifs apparaissent déjà très élevés : $25 \%$ des femmes et $18 \%$ des hommes du groupe de référence sont concernés par un score au PHQ-4 égal ou supérieur à 6 (Tableau 2). Ils sont jusqu'à trois fois supérieurs à ceux observés en population générale en Allemagne avec la même échelle : le PHQ-4 (Löwe et al., 2010). L'absence de différence statistiquement significative entre les groupes étudiés implique qu'il n'y a pas d'effet notable de l'infection VIH sur les symptômes anxieux et dépressifs dans cette population.

Tableau 2 : Liens transnationaux et niveau de symptômes anxieux et dépressifs modérés à sévères des femmes et des hommes selon le groupe d'étude

\begin{tabular}{|c|c|c|c|c|c|c|c|c|}
\hline \multirow[b]{3}{*}{$\begin{array}{l}\text { Avoir un enfant de } \\
\text { moins de } 18 \text { ans } \\
\text { dans le pays } \\
\text { d'origine/autre pays }\end{array}$} & \multicolumn{4}{|c|}{ Femmes } & \multicolumn{4}{|c|}{ Hommes } \\
\hline & $\begin{array}{l}\text { Groupe de } \\
\text { référence }\end{array}$ & VIH & Hépatite $B$ & & $\begin{array}{l}\text { Groupe de } \\
\text { référence }\end{array}$ & VIH & Hépatite B & \\
\hline & 15,5 & 19,6 & 18,6 & 0,444 & 32,5 & 37,8 & 33,5 & 0,527 \\
\hline $\begin{array}{l}\text { Pouvoir compter } \\
\text { sur quelqu'un dans } \\
\text { le pays d'origine }\end{array}$ & 77,9 & 66,0 & 70,8 & $<0,001$ & 74,8 & 67,1 & 72,7 & 0,123 \\
\hline \multicolumn{9}{|c|}{ Pouvoir compter sur quelqu'un } \\
\hline $\begin{array}{l}\text { En France et dans } \\
\text { le pays d'origine }\end{array}$ & 71,8 & 53,8 & 67,0 & $<0,001$ & 65,2 & 57,8 & 65,4 & 0,116 \\
\hline $\begin{array}{l}\text { Uniquement en } \\
\text { France }\end{array}$ & 13,9 & 23,5 & 16,5 & & 11,6 & 23,9 & 17,6 & \\
\hline $\begin{array}{l}\text { Uniquement dans } \\
\text { le pays d'origine }\end{array}$ & 6,1 & 12,2 & 3,8 & & 9,6 & 9,3 & 7,3 & \\
\hline $\begin{array}{l}\text { N'avoir personne } \\
\text { sur qui compter }\end{array}$ & 8,2 & 10,5 & 12,7 & & 13,6 & 8,9 & 9,7 & \\
\hline \multicolumn{9}{|c|}{ Symptômes anxieux et dépressifs } \\
\hline $\begin{array}{l}\text { Modérés à sévères } \\
(\mathrm{PHO}-4 \geq 6)\end{array}$ & 24,5 & 31,4 & 21,7 & 0,114 & 17,5 & 19,6 & 19,7 & 0,769 \\
\hline
\end{tabular}

Source : Enquête ANRS-Parcours 2012-2013. 


\section{Les liens entre ici et là-bas et la santé mentale}

Nous évaluons à présent le rôle des liens transnationaux sur les symptômes d'anxiété et de dépression à l'aide d'une régression logistique. Il s'agit d'estimer si "toutes choses égales par ailleurs" nous observons toujours une association statistique entre les indicateurs définis pour mesurer les liens transnationaux et ceux définis pour évaluer la présence de symptômes anxieux et dépressifs. La formulation "toutes choses égales par ailleurs" signifie que les associations observées tiennent compte d'un ensemble de caractéristiques individuelles telles que l'âge, le niveau d'étude, la situation professionnelle, administrative, le temps écoulé depuis la migration, la raison de la migration. Le modèle statistique est alors dit "ajusté» (Tableau 3, colonne "ORa»).

L'enquête Parcours indique qu'il n'y a pas de lien «toutes choses égales par ailleurs" entre la séparation d'avec un enfant et les symptômes anxieux et dépressifs, ni pour les femmes, ni pour les hommes (ORa $=1,0[0,6-1,6], \mathrm{ORa}=$ $1,3[0,9-1,9]$, respectivement, Tableau 3). La situation sociale et administrative des personnes est plus difficile pour les personnes qui sont séparées d'un de leurs enfants. C'est cette situation, notamment l'absence de titre de séjour stable qui altère la santé mentale (Pannetier et al., 2017). Lorsque I'on "ajuste" le modèle, autrement dit lorsque l'on tient compte de cette différence, la parentalité transnationale n'est pas associée aux symptômes anxio-dépressifs. Pouvoir faire venir l'enfant, même ponctuellement ou pouvoir faire des allers et retours est certainement très important pour prévenir les tensions mentales liées à la séparation. Ces résultats permettent de nuancer les représentations/les discours qui font de la séparation physique $d^{\prime}$ avec un enfant nécessairement une source de mal-être, et ce, surtout pour les femmes. Le fait qu'un enfant réside à l'étranger peut exposer à des attitudes stigmatisantes. Les modèles de maternité de la société française contemporaine produisent des injonctions différentes des modèles de maternité des profils dominants des sociétés d'origine des populations étudiées où les enfants circulent plus entre les membres de la parenté. En outre, des études récentes montrent qu'en cas de séparation (par une migration) des stratégies garantissent le maintien de la relation parents-enfants par-delà des frontières et atténuent les tensions liées à cette séparation, dont le maintien d'un sentiment d'appartenance. La qualité des relations entre les enfants, les parents et les personnes qui ont en garde les enfants joue également un rôle important, de même que les normes socioculturelles qui régissent la mobilité/circulation et l'éducation des enfants (Poeze et al., 2016; Berckmoes et Mazzucato, 2018). D'autres recherches sont néanmoins nécessaires pour approfondir et éclairer ces résultats.

Par ailleurs, l'idéal social du regroupement familial n'est pas toujours possible, les parents migrants peuvent avoir des charges de travail importantes et des conditions de vie pas toujours propices à la venue de leurs enfants. Ils peuvent craindre également l'isolement de ces derniers dans des contextes ou leur marginalisation sociale est probable (Yépez et al., 2011). La possibilité de se retrouver des membres d'une famille transnationale est également très liée aux politiques migratoires, car les contraintes juridiques influencent les conditions de réunification même temporaire des familles (Beauchemin et al., 2014; Mazzucato et al., 2015). La stabilité matérielle et administrative permet la circulation des membres de la famille, limitant ainsi la durée des séparations, source potentielle de souffrance et d'amenuisement ou de rupture des liens. 
Tableau 3 : Liens transnationaux et symptômes anxieux et dépressifs modérés à sévères (score au $\mathrm{PHO}-4 \geq 6$ ), par sexe ${ }^{10}$

\begin{tabular}{|c|c|c|c|c|}
\hline & \multicolumn{2}{|c|}{ Femmes } & \multicolumn{2}{|c|}{ Hommes } \\
\hline & \multicolumn{2}{|c|}{$\mathrm{N}=1189$} & \multicolumn{2}{|c|}{$N=1253$} \\
\hline & OR & ORa & OR & ORa \\
\hline \multicolumn{5}{|c|}{ Liens de solidarité avec des personnes du pays d'origine et en France } \\
\hline $\begin{array}{l}\text { Avoir quelqu'un sur qui compter en } \\
\text { France et dans le pays d'origine }\end{array}$ & $0,4 * * *[0,2-0,6]$ & $0,5 *[0,3-1,0]$ & $0,3 * * *[0,2-0,5]$ & $0,5 * *[0,3-0,8]$ \\
\hline $\begin{array}{l}\text { Avoir quelqu'un sur qui compter } \\
\text { uniquement en France }\end{array}$ & $0,5^{*}[0,3-0,9]$ & $0,7[0,4-1,2]$ & $0,6[0,4-1,1]$ & $1,0[0,6-1,7]$ \\
\hline $\begin{array}{l}\text { Avoir quelqu'un sur qui compter } \\
\text { uniquement dans le pays d'origine }\end{array}$ & $1,2[0,6-2,8]$ & $1,3[0,5-3,0]$ & $1,2[0.6-2,2]$ & $1,0[0,6-1,9]$ \\
\hline N'avoir personne sur qui compter & Réf. & Réf. & Réf. & Réf. \\
\hline \multicolumn{5}{|c|}{ Avoir un enfant de moins de $\mathbf{1 8}$ ans dans le pays d'origine/autre pays } \\
\hline Oui & $1,9 * *[1,2-2,8]$ & $1.0[0,6-1,6]$ & $1,8^{* *}[1,2-2,7]$ & $1,3[0,9-1,9]$ \\
\hline Non & Réf. & Réf. & Réf. & Réf. \\
\hline
\end{tabular}

Champ : Ensemble des personnes enquêtées.

Note : Les résultats ont été obtenus à l'aide de régressions logistiques ajustées sur l'âge, le niveau d'étude, la pratique religieuse, le fait d'être en couple, le statut d'occupation, la durée de séjour en France, la raison de la migration, la situation administrative, le fait d'avoir vécu dans la rue et le fait d'avoir subi un rapport forcé http://www.ceped.org/parcours : chapitre 13 annexes.

Lecture : Un coefficient supérieur (respectivement inférieur) à 1 et statistiquement significatif indique que la probabilité de connaitre des symptômes anxieux et dépressifs est supérieure (respectivement inférieurs) par rapport à la modalité de référence.

Légende : Significativité à $*: 5 \%{ }^{* *}: 1 \%{ }^{* * *}: 0,1 \%$. Réf. : Situation de référence. OR :

Odds ratio calculés par régression logistique et ajustés sur le groupe d'étude. ORa : Odds ratios calculés par régression logistique et ajustés sur le groupe d'étude et toutes les autres variables incluses dans le modèle.

Source : Enquête ANRS-Parcours 2012-2013.

Par ailleurs, l'existence d'un réseau de soutien à la fois "ici et là-bas" a un effet positif sur la santé mentale des personnes, en comparaison aux personnes qui déclarent n'avoir personne sur qui compter. La probabilité d'avoir des symptômes d'anxiété et de dépression est plus faible pour les femmes et les hommes qui déclarent pouvoir compter sur quelqu'un à la fois dans le pays d'origine et en France en comparaison aux personnes qui déclarent n'avoir personne sur qui compter $(\mathrm{ORa}=0,5[0,3-1,0], \mathrm{ORa}=0,5[0,3-0,8]$, respectivement, Tableau 3). En revanche, avoir quelqu'un sur qui compter uniquement dans le pays d'origine ou uniquement en France n'est pas associé à un moindre risque de connaitre des symptômes anxieux et dépressifs après ajustement sur

10 Les résultats présentés tiennent compte de l'âge et du nombre d'années passées en France. Ils tiennent compte également des effets liés à la pathologie. Par ailleurs, les analyses ont été stratifiées sur le temps passé en France, les résultats sont inchangés. Les études ont été stratifiées par pathologies et les résultats sont stables. 
les autres facteurs connus de la dépression (Tableau 3). Ces résultats corroborent ceux d'une étude qualitative réalisée à Detroit, aux États-Unis, auprès des femmes mexicaines montrant que si le réseau de soutien local entre les femmes mexicaines et leurs proches aux États-Unis favorise leur installation dans le pays d'immigration, le soutien des parents, des frères et sœurs vivant au Mexique aide ces femmes à faire face à un ensemble de difficultés vécues dans le pays de résidence et leur apporte " an alternative space of belonging" (Viruell-Fuentes et Schulz, 2009) permettant de rompre avec l'isolement. La similitude des résultats entre les femmes et hommes tient possiblement au rapprochement des profils migratoires entre ces derniers (Beauchemin et al., 2013). Les migrations dites "autonomes" (c'est-à-dire celles autres que reliées au regroupement familial) des femmes augmentent. Le temps nécessaire afin d'obtenir un minimum de stabilité $^{11}$ est proche entre les femmes et les hommes (Gosselin et al., 2016). Cette stabilité nécessite sans doute le soutien des proches à la fois en France et dans leur pays d'origine.

\section{Conclusion}

La question des liens transnationaux ainsi que de leurs rôles sur la santé mentale des personnes migrantes reste un champ de recherche à construire, il faudrait pouvoir aborder ces liens de façon fine et dans leurs multiples facettes et sources de soutien et/ou de conflits. Seuls deux aspects des liens transnationaux (le soutien transnational et la parentalité transnationale) ont été questionnés ici, l'enquête utilisée n'ayant pas vocation à questionner les tensions mentales liées aux pratiques transnationales ou à la famille transnationale. Nos résultats soulignent néanmoins que les pratiques transnationales ne sont pas bilatérales, les échanges peuvent être réciproques et contribuer à préserver des souffrances psychiques causées par un contexte sociopolitique marqué par des conditions d'installation de plus en plus difficiles en France. En effet, les personnes migrantes qui bénéficient d'un double réseau de soutien, "ici" en France et "là-bas" dans le pays d'origine, présentent un risque moindre d'avoir des symptômes anxieux et dépressifs. Ces résultats permettent de contribuer aux débats sur l'effet des liens que les personnes migrantes entretiennent avec leur pays d'origine. Les pratiques transnationales peuvent être perçues comme un fardeau pour des personnes immigrées inscrites dans des logiques de dettes matérielles, sociales ou affectives vis-à-vis de leur famille. Cependant notre étude montre que le soutien (affectif, social ou matériel) peut également venir de "là-bas" et qu'il représente, en combinaison avec un réseau de soutien "ici», une ressource, ou une forme de résilience face à un ensemble de difficultés. L'étude révèle également que la séparation d'avec un enfant n'entraine pas nécessairement un risque plus élevé de connaitre des symptômes anxieux et dépressifs. Ce qui permet de nuancer les discours qui font de la séparation d'un enfant nécessairement une source de mal-être, et ce, surtout pour les femmes. Ces résultats demanderaient à être consolidés par d'autres recherches, il faudrait pouvoir aller plus loin dans l'exploration des formes de résiliences qui

11 La séquence d'installation diffère selon le sexe, alors que les hommes entament leur processus d'installation par le travail, les femmes, plus souvent venues pour rejoindre un conjoint ou de la famille, débutent le leur par l'accès à un logement personnel puis par l'accès au titre de séjour (Gosselin et al., 2016). 
permettent de faire face aux tensions mentales induites par la migration (dont la parentalité transnationale) sans oublier que celles-ci dépendent étroitement des politiques migratoires et de la position des personnes dans les rapports sociaux.

\section{Références bibliographiques}

Alcántara Carmela, Chen Chih-Nan and Alegría Margarita (2015) Transnational ties and past-year major depressive episodes among Latino immigrants, Cultural Diversity \& Ethnic Minority Psychology, 21 (3), pp. 486-495.

Antoine Philippe, Lelièvre Eva et le Groupe de Réflexion sur I'Approche Biographique (2006) États flous et trajectoires complexes : observation, modélisation, interprétation, Paris, INED, 301 p.

Audebert Cédric, Lacroix Thomas et Ma Mung Emmanuel (2018) La famille transnationale dans les recherches sur les migrations, in Christophe Imbert, Eva Lelièvre et David Lessault Dirs., Mobilités, territoires et liens familiaux, Paris, INED, pp. 73-92.

Beauchemin Cris, Borrel Catherine et Régnard Corinne (2013) Les immigrés en France : en majorité des femmes, Population \& Sociétés, 502, 4 p.

Beauchemin Cris, Hamel Christelle, Lesné Maud et Simon Patrick (2010) Les discriminations : une question de minorités visibles, Population \& Sociétés, 466, $4 \mathrm{p}$.

Beauchemin Cris, Hamel Christelle et Simon Patrick (2015a) Trajectoires et origines. Enquête sur la diversité des populations en France, Paris, INED, 623 p.

Beauchemin Cris, Lagrange Hugues et Safi Mirna (2015b) Liens transnationaux et intégration : entre ici et là-bas, in Cris Beauchemin, Christelle Hamel et Patrick Simon Éds., Trajectoires et origines. Enquête sur la diversité des populations en France, Paris, INED, pp. 87-116.

Beauchemin Cris, Nappa Jocelyn, Schoumaker Bruno, Baizan Pau, González】 Ferrer Amparo, Caarls Kim and Mazzucato Valentina (2014) Reunifying Versus Living Apart Together Across Borders: A Comparative Analysis of sub-Saharan Migration to Europe, International Migration Review, 49 (1), pp. 173-199.

Berchet Caroline et Jusot Florence (2010) L'état de santé des migrants de première et de seconde génération en France, Revue économique, 61 (6), pp. 1075-1098.

Berckmoes Lidewyde and Mazzucato Valentina (2018) Resilience among Nigerian transnational parents in the Netherlands: a strength based approach to migration and transnational parenting, Global Networks: a Journal of Transnational Affairs, $18(4)$, pp. 589-607.

Bhui Kamaldeep, Stansfeld Stephen, McKenzie Kwame, Karlsen Saffron, Nazroo James and Weich Scott (2005) Racial/ethnic discrimination and common mental disorders among workers: findings from the EMPIRIC Study of Ethnic Minority Groups in the United Kingdom, American Journal of Public Health, 95 (3), pp. 496-501.

Beutel Manfred, Jünger Claus, Klein Eva, Wild Philipp, Lackner Karl, Blettner Maria, Mita Banerjee, Michal Matthias, Wiltink Jörg and Brähler Elmar (2016) Depression, anxiety and suicidal ideation among $1 \mathrm{st}$ and 2 nd generation migrants: results from the Gutenberg health study, BMC Psychiatry, 16, p. 288. 
Bredeloup Sylvie (2008) L'aventurier, une figure de la migration africaine, Cahiers internationaux de sociologie, 125, pp. 281-306.

Cazein Françoise, Pillonel Josiane, Le Strat Yann, Pinget Roselyne, Le Vu1 Stéphane, Brunet Sylvie, Thierry Damien, Brand Denys, Leclerc Marlène, Benyelles Lotfi, Da Costa1 Clara, Barin Francis et Lot Florence (2015) Découvertes de séropositivité VIH et sida. France, 2003-2013, Bull Epidémiol Hebd, 9-10, pp. 152-161.

Cebotari Victor, Mazzucato Valentina and Ernest Appiah (2018) A Longitudinal Analysis of Well-Being of Ghanaian Children in Transnational Families, Child development, 89 (5), pp. 1768-1785.

Couillet Maider (2010) Les Africains subsahariens vivant en France. Caractéristiques sociodémographiques et accès aux soins, Working Paper du CEPED, $99 \mathrm{p}$.

Desgrées du Loû Annabel et Lert France (dir.) (2017) Parcours. Parcours de vie et de santé des Africains immigrés en France, Paris, La Découverte, 360 p.

Desgrées du Loû Annabel, Pannetier Julie, Ravalihasy Andrainolo, Gosselin Anne, Supervie Virginie, Panjo Henri, Bajos Nathalie, Lert France, Lydié Nathalie and Dray-Spira Rosemary (2015) Sub-Saharan African migrants living with HIV acquired after migration, France, ANRS PARCOURS study, 2012 to 2013, Euro Surveillance: European Communicable Disease Bulletin, 20 (46).

Desgrées du Loû Annabel, Pannetier Julie, Ravalihasy Andrainolo, Le Guen Mireille, Gosselin Anne, Panjo Henri, Bajos Nathalie, Lert France, Lydié Nathalie and Dray-Spira Rosemary (2016) Is hardship during migration a determinant of HIV infection? Results from the ANRS PARCOURS study of sub-Saharan African migrants in France, AIDS (London, England), 30 (4), pp. 645-656.

Falcón Luis, Todorova Irina and Tucker Katherine (2009) Social support, life events, and psychological distress among the Puerto Rican population in the Boston area of the United States, Aging \& Mental Health, 13 (6), pp. 863-873.

Fassin Didier (2000) Les politiques de l'ethnopsychiatrie La psyché africaine, des colonies africaines aux banlieues parisiennes, L'Homme, 153, pp. 231-250.

Fassin Didier et Fassin Éric (2006) De la question sociale à la question raciale? Représenter la société française, Paris, La Découverte, 280 p.

Findley Sally (1999) La famille africaine et la migration, La famille africaine, Politiques démographiques et développement, Paris, Karthala, pp. 153-193.

Fouron George and Glick Schiller Nina (2002) The Generation of Identity: Redefining the Second Generation Within a Transnational Social Field, in Peggy Levitt and Mary Waters Eds., The Changing Face of Home. The Transnational Lifes of Second Generation, New York, Russel Sage, pp. 168-208.

Glick Schiller Nina, Basch Linda and Szanton Blanc Cristina (1995) From Immigrant to Transmigrant: Theorizing Transnational Migration, Anthropological Quarterly, 68 (1), pp. 48-63.

Gosselin Anne, Desgrées du Loû Annabel, Lelièvre Eva, Lert France, Dray-Spira Rosemary et Lydié Nathalie (2016) Migrants subsahariens : combien de temps leur faut-il pour s'installer en France?, Population \& Sociétés, 533, 4 p.

Haagsma Karlijn, Mazzucato Valentina and Dito Bilisuma (2015) Transnational families and the subjective well-being of migrant parents: Angolan and Nigerian parents in the Netherlands, Ethnic and Racial Studies, 38 (15), pp. 2652-2671. 
Hamel Christelle et Moisy Murielle (2012) Migrations, conditions de vie et santé en France à partir de I'enquête Trajectoires et origines, Bull Epidémiol Hebd, 2-3-4, pp. 21-24.

Ichou Mathieu, Goujon Anne et I'équipe de I'enquête DiPAS (2017) Le niveau d'instruction des immigrés : varié et souvent plus élevé que dans les pays d'origine, Population \& Sociétés, 541, 4 p.

INSEE (2011) Étrangers/Immigrés - Séries issues des recensements de la population - RP2011.

Kroenke Kurt, Spitzer Robert, Williams Janet and Löwe Bernd (2009) An ultrabrief screening scale for anxiety and depression: the PHQ-4, Psychosomatics, 50 (6), pp. 613-621.

Lessault David et Beauchemin Cris (2009) Les migrations d'Afrique subsaharienne en Europe : un essor encore limité, Population \& Sociétés, 452, 4 p.

Levecque Katia, Lodewyckx Ina and Vranken Jan (2007) Depression and generalised anxiety in the general population in Belgium: a comparison between native and immigrant groups, Journal of Affective Disorders, 97 (1-3), pp. 229-239.

Levitt, Peggy and Jaworsky Nadya (2007) Transnational migration studies: past developments and future trends, Annual Review of Sociology, 33, pp. 129-156.

Löwe Bernd, Wahl Inka, Rose Matthias, Spitzer Carsten, Glaesmer Heide, Wingenfeld Katja, Schneider Antonius and Brähler Elmar (2010) A 4-item measure of depression and anxiety: Validation and standardization of the Patient Health Questionnaire-4 (PHQ-4) in the general population, Journal of Affective Disorders, 122 (1-2), pp. 86-95.

Ly Mouhamed, Petit Véronique et Pizzolato Giulia (2014) La migration internationale face à la santé mentale au Sénégal : récits, discours et imaginaires, in Cécile Canut et Catherine Mazauric Éds., Les mots de la migration. Mise en récits et en images des migrations transafricaines, Paris, Le cavalier bleu, pp. 221-239.

Massey Douglas, Arango Joaquin, Hugo Graeme, Kouaouci Ali, Pellegrino Adela and Taylor Edward (1993) Theories of International Migration: A Review and Appraisal, Population and Development Review, 19 (3), pp. 431-466.

Mazzucato Valentina, Schans Djamila, Caarls Kim and Beauchemin Cris (2015) Transnational Families Between Africa and Europe, International Migration Review, 49 (1), pp. 142-172.

Meurs Dominique, Lhommeau Bertrand et Okba Mahrez (2015) Emplois, salaires et mobilité intergénérationnelle, in Cris Beauchemin, Christelle Hamel et Patrick Simon Éds., Trajectoires et origines, Enquête sur la diversité des populations en France, Paris, INED, pp. 233-263.

Meurs Dominique, Pailhé Ariane et Simon Patrick (2007) Persistance des inégalités entre générations liées à l'immigration : I'accès à l'emploi des immigrés et de leurs descendants en France, Population, 61 (5), pp. 763-801.

Missinne Sarah and Bracke Piet (2012) Depressive symptoms among immigrants and ethnic minorities: a population based study in 23 European countries, Social Psychiatry and Psychiatric Epidemiology, 47 (1), pp. 97-109.

Ndiaye Pape (2008) La Condition Noire. Essai sur une minorité française, Paris, Calmann-Lévy, 436 p. 
Ornelas India and Perreira Krista (2011) The role of migration in the development of depressive symptoms among Latino immigrant parents in the USA, Social Science \& Medicine (1982), 73 (8), pp. 1169-1177.

Pan Ké Shon Jean-Louis et Duthé Géraldine (2013) Trente ans de solitude... et de dépression, Revue française de sociologie, 54 (2), pp. 225-261.

Pannetier Julie, Desgrées du Loû Annabel et Lert France (2017) Santé mentale et parcours migratoires : symptômes d'anxiété et de dépression, in Annabel Desgrées du Loû et France Lert Éds., Parcours de vie et de santé des Africains immigrés en France, Paris, La Découverte, pp. 287-298.

Poeze Martijn, Dankyi Ernestina and Mazzucato Valentina (2016) Navigating transnational childcare relationships: Migrant parents and their children's caregivers in the origin country, Global Networks: a Journal of Transnational Affairs, 17 (1), pp. 111-129.

Portes Alejandro (1999), La mondialisation par le bas, l'émergence des communautés transnationales, Actes de la recherche en sciences sociales, 129, pp. 15-25.

Quiminal Catherine (1991) Gens d'ici, gens d'ailleurs : migrations Soninké et transformations villageoises, Paris, Christian Bourgeois, 222 p.

Razy Élodie et Baby-Collin Virginie (2011) La famille transnationale dans tous ses états, Autrepart, 57-58 (1), pp. 7-22.

Retchman Richard (2000) De la psychiatrie des migrants au culturalisme des ethnopsychiatres, Hommes et Migrations, 1225, pp. 46-61.

Sargent Caroline and Larchanché Stéphanie (2016) Transnational healthcare circuits: Managing therapy among immigrants in France and Kin in West Africa, in Jennifer Cole and Christian Groes Eds., Affective Circuits: African Migrations to Europe and the Pursuit of Social Regeneration, Chicago, University of Chicago Press, $352 \mathrm{p}$.

Sayad Abdelmalek (2006) L'immigration ou les paradoxes de l'altérité. 1. L'illusion du provisoire, Paris, Raisons d'agir, $218 \mathrm{p}$.

Timera Mahamet et Garnier Julie (2010) Les Africains en France, Hommes et migrations, 1286-1287, pp. 24-35.

Torres Jacqueline (2013) Cross-border ties and self-rated health status for young Latino adults in Southern California, Social Science \& Medicine (1982), 81, pp. 79-86.

Torres Jacqueline, Alcántara Carmela, Rudolph Kara and Viruell-Fuentes Edna (2016a) Cross-borderTies as Sources of Risk and Resilience: Do Cross-borderTies Moderate the Relationship between Migration-related Stress and Psychological Distress for Latino Migrants in the United States?, Journal of Health and Social Behavior, 57 (4), pp. 436-452.

Torres Jacqueline, Lee Anne, González Hector, Garcia Lorena and Haan Mary (2016b) Longitudinal analysis of cross-border ties and depression for Latino adults, Social Science \& Medicine (1982), 160, pp. 111-119.

Tortelli Andrea, Morgan Craig, Szoke Andrei, Nascimento Andreia, Skurnik Norbert, de Caussade Erik, Fain-Donabedian Edith, Fridja Flora, Henry Mehedi, Ezembe Ferdinand and Murray Robin (2014) Different rates of first admissions for psychosis in migrant groups in Paris', Social Psychiatry and Psychiatric Epidemiology, 49 (7), pp. 1103-1109. 
Veïsse Arnaud, Wolmark Laure, Revault Pascal, Giacopelli Maud, Bamberger Muriel et Zlatanova Zornitza (2017) Violence, vulnérabilité sociale et troubles psychiques chez les migrants/exilés, Bull Epidémiol Hebd, 19-20, pp. 405-414.

Viruell-Fuentes Edna and Schulz Amy (2009) Toward a dynamic conceptualization of social ties and context: implications for understanding immigrant and Latino health, American Journal of Public Health, 99 (12), pp. 2167-2175.

Wang Simeng (2017) Illusions et souffrances. Les migrants chinois à Paris, Paris, Rue d'Ulm, 220 p.

Wang Simeng (2016) " Aidez-nous à comprendre vos Chinois! ". Conditions de possibilités de la légitimation du sociologue en milieu psychiatrique, Genèses, 105 (4), pp. 141-156.

Yépez Isabel, Ledo Carmen et Mirko Marzadro (2011) «Si tu veux que je reste ici, il faut que tu t'occupes de nos enfants! ». Migration et maternité transnationale entre Cochabamba (Bolivie) et Bergame (Italie), Autrepart, 57-58 (1), pp. 199-213. 


\section{Julie Pannetier}

\section{Liens transnationaux et santé mentale : de la nécessité du lien entre ici et là-bas? Le cas des migrations africaines en Île-de-France}

Les personnes migrantes peuvent être inscrites dans des logiques de dettes matérielles, sociales ou affectives vis-à-vis de leurs proches restés au pays, ainsi la question du coût des pratiques transnationales (notamment des transferts monétaires) pour leur santé a été soulignée. Cependant, peu d'études ont porté sur l'effet protecteur du soutien (matériel, social, affectif) des proches résidant dans le pays d'origine sur leur santé mentale. L'enquête Parcours a été menée en 2012-2013 dans soixante-quatorze structures de soin en île-de-France auprès de 2468 personnes migrantes originaires d'Afrique. Cette enquête permet, à travers différents indicateurs, d'interroger le rôle de la parentalité transnationale et d'un soutien transnational sur la santé mentale des personnes migrantes. Cette recherche montre notamment que les femmes et les hommes qui déclarent pouvoir compter sur leurs proches, parents ou amis, à la fois dans le pays d'origine et en France présentent les niveaux d'anxiété et de dépression les plus bas. Un double réseau de soutien à la fois "ici et là-bas" aurait un effet protecteur sur la santé mentale des personnes migrantes.

\section{Transnational Ties and Mental Health: A Need for a Link between Here and There? The Case of African Migrations in France}

Migrants may be embedded in logics of material, social or emotional debts to relatives in the country of origin, therefore the emotional cost of transnational practices (espacially remittances) has been underlined. However, few studies have focused on the protective effect of transnational support (material, social, emotional) from relatives in migrant mental health. The Parcours survey was conducted in 2012-2013 in seventy-four health care facilities in the Parisian region among 2.468 migrants from Sub-Saharan Africa. Through different indicators, this survey examines the role of transnational parenthood and transnational support on migrant mental health. This study shows that women and men who claim to be able to rely on relatives or friends, both in the country of origin and in France, have lower levels of anxiety and depression. A support network from both "here and there" may have a protective effect on the mental health of migrants.

\section{Vínculos trasnacionales y salud mental: ¿necesidad de una conexión aquí y allá? El caso de las migraciones africanas en Francia}

Las personas migrantes están generalmente inscritas dentro de una lógica de deudas materiales, sociales o afectivas con sus familiares, y varios estudios han puesto de manifiesto el costo de estas prácticas para su salud. Sin embargo, pocos estudios han abordado el efecto protector en la salud mental del sostén (material, social, afectivo) brindado por los allegados desde el país de origen. El estudio Parcours, realizado con personas emigradas de África subsahariana muestra que aquellos que declararon poder contar con sus allegados, padres o amigos, tanto en el país de origen como en Francia, presentan niveles más bajos de ansiedad o depresión. De esta forma, la existencia de una red de apoyo tanto "aquí como allá» tiene un efecto protector en la salud mental de las personas migrantes de África subsahariana. 